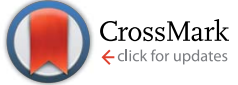

Cite this: RSC Adv., 2016, 6, 83217

Received 18th July 2016

Accepted 22nd August 2016

DOI: 10.1039/c6ra18317d

www.rsc.org/advances

\section{Control of the size and density of ZnO-nanorods grown onto graphene nanoplatelets in aqueous suspensions $\dagger$}

\author{
C. R. Chandraiahgari, ${ }^{\text {ab }}$ G. De Bellis, ${ }^{\text {ab }}$ S. K. Balijepalli, ${ }^{c}$ S. Kaciulis, ${ }^{c}$ P. Ballirano, ${ }^{\text {bd }}$ \\ A. Migliori, ${ }^{e}$ V. Morandi, ${ }^{e}$ L. Caneve, ${ }^{f}$ F. Sarto ${ }^{f}$ and M. S. Sarto ${ }^{\star a b}$
}

\begin{abstract}
Zinc oxide nanorods (ZnO-NRs) with high density and chemical purity were grown onto unsupported graphene nanoplatelets (GNPs) in aqueous suspensions, using two different growth approaches namely: a hydrothermal method and ultrasonic probe sonication. The size and density of the ZnO-nanorods grown onto graphene nanoplatelets were controlled through seed layer deposition and through the proper setting of the process parameters, in particular through the control of the fluidodynamics of the colloidal suspension during the growth. The highest growth density of the $\mathrm{ZnO}$ nanorods having a diameter of $\sim 45$ $\mathrm{nm}$ was obtained onto GNPs seeded by the probe sonication method and through the hydrothermal method in dynamic conditions. XRD and XPS investigations confirmed that all produced ZnO-GNP composites are characterized by high crystallinity and purity, although solution dynamics affected their UV luminescence. The proposed approaches enable the controlled high-density growth of crystalline $\mathrm{ZnO}$ NRs onto GNPs in an aqueous suspension, at a low cost, and are suitable for large scale production.
\end{abstract}

\section{Introduction}

Graphene, owing to its exceptional electrical, thermal, and mechanical properties, has been widely explored in a variety of fields, such as electronics, sensors, catalysis, drug delivery, solar cells, energy harvesting and storage systems. ${ }^{\mathbf{1 , 2}}$ Decoration or functionalization of pristine graphene with inorganic nanostructures is a way to bring additional functionality to it. ${ }^{3}$ Recently, hybrid systems made of graphene-based materials and ZnO-nanostructures have been investigated for their potentiality as a new class of multifunctional nanomaterials exploiting the salient features of graphene and $\mathrm{ZnO}^{4-8}$

Hybrid nanostructures or composites made of $\mathrm{ZnO}$ and graphene have resulted in multifunctional and enhanced properties such as high UV sensing capabilities, excellent field emission, ultrafast nonlinear optical switching, gas sensing, improved photocatalytic activity, and piezoelectricity. ${ }^{5-10}$ Such

\footnotetext{
${ }^{a}$ Department of Astronautics, Electrical and Energetics Engineering, Sapienza University of Rome, via Eudossiana 18, Rome 00184, Italy. E-mail: c. chandraiahgari@uniroma1.it; mariasabrina.sarto@uniroma1.it

${ }^{b}$ Research Center for Nanotechnology Applied to Engineering of Sapienza (CNIS), SNNLab, Sapienza University of Rome, Piazzale Aldo Moro, 5, Rome 00185, Italy ${ }^{c}$ CNR - ISMN, P.O. Box 10, 00015 Monterotondo Stazione, Rome, Italy

${ }^{d}$ Department of Earth Sciences, Sapienza University of Rome, Piazzale Aldo Moro 5, Rome 00185, Italy

${ }^{e}$ CNR - IMM Section of Bologna, via Gobetti 101, Bologna 40129, Italy ${ }^{f}$ ENEA, Centro Ricerche Frascati, Via Enrico Fermi 45, Frascati 00044, Italy

$\dagger$ Electronic supplementary information (ESI) available. See DOI: 10.1039/c6ra18317d
}

enhanced properties mainly arise from the combination of the superior electrical properties of the carbon-based materials with the optical properties and polarity of ZnO. ${ }^{11,12}$ Several studies demonstrated that shape, size, concentration and distribution of the nanostructures in a composite are greatly affecting the functional properties of the final material. ${ }^{13-15}$ Therefore, the control of shape, size and morphology through the proper setting of the growth conditions plays a crucial role in tuning the functional properties.

$\mathrm{ZnO}$ crystals have a noncentral symmetric wurtzite structure and are composed of closely packed $\mathrm{O}_{2}{ }^{-}$and $\mathrm{Zn}^{2+}$ layers piled alternatively along the c-axis, producing positively charged $\mathrm{Zn}$ terminated [0001] polar surfaces and negatively charged O-terminated [0001] polar surfaces. ${ }^{16}$ ZnO-nanocrystals with varying surface configurations and polarities display different optical and electronic properties. However, synthesis of $\mathrm{ZnO}$ nanostructures (i.e. $c$-axis oriented nanowires or $a$-axis oriented nanobelts ${ }^{17}$ ) is very sensitive to the growth conditions ${ }^{18-20}$ and, consequently, special care must be paid in order to assure reproducibility and controllability.

Recently, interesting progresses have been made towards the fabrication of $\mathrm{ZnO}$-graphene hybrid nanostructures by using different techniques, such as: chemical vapor deposition (CVD) of $\mathrm{ZnO}$ nanostructures on CVD-grown graphene; synthesis in liquid solution of ZnO-NRs onto graphene flakes transferred via scotch-tape; synthesis in liquid solution of ZnO-NRs on CVDgrown graphene; synthesis in liquid solution of ZnO-NRs onto chemically-reduced graphene oxide (RGO).10,21-23 In these studies, the functional properties of the hybrid nanostructures 
were controlled through the synthesis methods. Various process parameters such as growth solution concentration, $\mathrm{pH}$ and temperature, tilt position and seed deposition affect the controlled growth of nanostructures. ${ }^{23} \mathrm{~J}$. Ding et al. $(2015),{ }^{21} \mathrm{Y}$. Kim et al. (2011) ${ }^{23}$ and M. Khenfouch et al. (2014) $)^{24}$ have grown ZnO-NRs through hydrothermal method onto supported fewlayer-graphene flakes and unsupported RGO without the use of a seed layer: this approach resulted in low density and poor homogeneity of the growth. Differently, R. Chung et al. (2013) ${ }^{22}$ and D. Shin et al. $(2014)^{5}$ deposited a seed layer either through electron-gun evaporation or through spin-coating onto supported CVD-grown graphene, and achieved a high-density growth, thus demonstrating the importance of seed layer. J. Fan et al. $(2015)^{25}$ deposited the seed layer by spin-coating onto supported monolayer graphene, and obtained high-density growth of twinned structures and microrod arrays, although clearly lacking in homogeneity and in morphology control.

It should be highlighted that in most of these studies, $\mathrm{ZnO}$ nanostructures were grown onto graphene supported on a substrate, so that decoration or functionalization was typically achieved only over the exposed surface of graphene. Moreover, it has not been shown, how different hydrodynamic conditions of the colloidal suspension during the growth enable the control of the density and shape of the $\mathrm{ZnO}$ nanostructures.

In this paper, we report the synthesis of $\mathrm{ZnO}$-nanostructures with controlled shape and density onto unsupported multilayergraphene flakes (also known as graphene nanoplatelets GNPs $^{2}$ ), using a seed layer, through aqueous solution-based lowtemperature approaches. ${ }^{\mathbf{2 6 , 2 7}}$ In particular, we investigate the role that the hydrodynamic conditions of the growth suspension have on the morphology, density, crystallinity and photoluminescence properties of ZnO-NR decorated GNPs (ZNGs). General models for particle nucleation and growth by hydrothermal method suggest that atoms fluctuating in liquid phase may collide and stick to existing seeds and/or already formed agglomerates. The so-formed clusters will remain stable if the energy gain associated to solid formation is higher than the energy cost of building the solid/liquid interface. ${ }^{28}$ Changing the hydrodynamic growth conditions from static to dynamic regime leads to an increase of the energy gain associated to solid formation, due to the increased concentration of the (precipitating) ions in the supersaturated phase, which may favour smaller nanostructures to become stable.

Therefore, in this study, we demonstrate that the proper setting of the seed-layer deposition process and of the hydrodynamic conditions during the ZnO-nanostructure growth lead to the production of ZNGs with controlled morphology, density, crystallinity and PL properties. To this purpose, FE-SEM, HRTEM, XRD, XPS, and PL techniques have been used to probe the properties of the produced ZNGs.

\section{Experimental}

\subsection{Materials}

All the chemicals were of reagent-grade and were used as received, without any further purification: graphite intercalation compound (GIC), zinc acetate dihydrate
$\left(\mathrm{Zn}\left(\mathrm{CH}_{3} \mathrm{COO}\right)_{2} \cdot 2 \mathrm{H}_{2} \mathrm{O}\right.$, Sigma-Aldrich, ACS reagent, $\left.\geq 98 \%\right)$, zinc nitrate hexahydrate $\left(\mathrm{Zn}\left(\mathrm{NO}_{3}\right)_{2} \cdot 6 \mathrm{H}_{2} \mathrm{O}\right.$, Acros Organics, 98\%), hexamethylenetetramine $\left(\mathrm{C}_{6} \mathrm{H}_{12} \mathrm{~N}_{4}\right.$, Fisher Scientific, $\left.\geq 99 \%\right)$, isopropanol $\left(\left(\mathrm{CH}_{3}\right)_{2} \mathrm{CHOH}\right.$, Sigma-Aldrich, ACS reagent, $\left.\geq 99 \%\right)$ and ethanol $\left(\mathrm{CH}_{3} \mathrm{CH}_{2} \mathrm{OH}\right.$, Fluka, ACS reagent, $\left.\geq 99 \%\right)$. The water, used in all the experiments, was deionized (DI) and had a resistivity of $18 \mathrm{M} \Omega \mathrm{cm}$.

\subsection{Methods}

Preparation of graphene nanoplatelets. GNPs were produced starting from GIC. ${ }^{2}$ Briefly, the GIC (having a declared mean particle size of $350 \mu \mathrm{m}$ and flake thickness of $50 \mu \mathrm{m}$ ) was thermally expanded in a muffle furnace for $5 \mathrm{~s}$ at $1150{ }^{\circ} \mathrm{C}$. An amount of $20 \mathrm{mg}$ of thermally expanded graphite was further subjected to a liquid phase exfoliation in $40 \mathrm{ml}$ of ethanol, through a sonotrode with ultrasonic probe (Sonics \& MaterialsVibracell VC505), operating in pulse mode (1 s for on- and off-phases) for $20 \mathrm{~min}$, at $70 \%$ of its maximum amplitude, and using a thermo-cryostat circulator in water bath set at $15^{\circ} \mathrm{C}$. The probe sonication resulted in the production of a colloidal suspension of GNPs, having thickness in the range of 2--10 nm and average lateral dimensions of $1-10 \mu \mathrm{m}$ as shown in ESI (page 2, Fig. S1†). Afterwards the solvent was removed by centrifugation.

Deposition of the seed layer onto GNPs suspended in aqueous solution. A seed solution was prepared by dissolving $0.005 \mathrm{M}$ of zinc acetate dehydrate in $40 \mathrm{ml}$ of isopropanol using magnetic bar stirring at room temperature. ${ }^{29}$ Prior to the deposition of the seed layer (SL), the obtained GNPs were refluxed in fresh isopropanol and the solvent was removed by centrifugation. GNPs were then dispersed into the seed solution through ultrasonication bath. Then, the suspension was magnetically stirred to obtain a uniform deposition of SL onto the GNPs. The suspension was then centrifuged and the precipitate was annealed inside a muffle furnace at $300{ }^{\circ} \mathrm{C}$ for 30 min, resulting in GNPs coated with quasi-spherical $\mathrm{ZnO}$ seed particles having an average diameter of $\sim 20 \mathrm{~nm}$.

The deposition of SL by probe sonication method was also performed using a very similar procedure, as described above, but replacing the step of magnetic stirring with a probe sonication. Probe sonication was performed for 15 min with constant ultrasound amplitude set at $45 \%$ of its maximum level. Finally, we obtained a suspension of GNPs coated with quasi spherical seed particles of $\mathrm{ZnO}$, having an average diameter of $\sim 15 \mathrm{~nm}$.

Growth of ZnO-NRs onto GNPs suspended in aqueous solution by hydrothermal method. ZnO-NRs were grown onto unsupported GNPs decorated with a SL, through the facile and cost-effective hydrothermal method. ${ }^{26}$ An aqueous growth solution was prepared by dissolving $0.02 \mathrm{M}$ equimolar ratios of zinc nitrate hexahydrate and hexamethylenetetramine together in $100 \mathrm{ml}$ of DI water. The solution was magnetically stirred at room temperature yielding a clear solution. Prior to the growth, the seeded GNPs were refluxed in fresh DI water. Next, the water was removed by centrifugation. The seeded GNPs were then suspended into the growth solution and a homogeneous 
dispersion was obtained by means of a ultrasonication bath. The suspension was then continuously stirred at $200 \mathrm{rpm}$ for 4.5 $\mathrm{h}$ on a hot plate (Heidolph MR-Hei Standard) using a bar magnet. During the reaction, the beaker was covered with an $\mathrm{Al}$ foil and the solution temperature was fixed at $60{ }^{\circ} \mathrm{C}$ using an automatic electronic temperature controller (EKT Hei-Con). After completion of the growth reaction, the suspension was centrifuged to remove the growth solution and washed twice in fresh DI water. The obtained precipitate was then dried in oven at $120{ }^{\circ} \mathrm{C}$ for $30 \mathrm{~min}$, thus obtaining ZnO-decorated GNPs. This procedure, hereinafter referred as hydrothermal method in dynamic condition, is denoted as HT1.

The influence of the continuous stirring of the seeded-GNP suspension during the $\mathrm{ZnO}-\mathrm{NR}$ growth reaction was assessed by implementing the aforementioned hydrothermal synthesis process keeping the suspension in a static condition (i.e. without the action of the mechanical stirring) at the same temperature of $60{ }^{\circ} \mathrm{C}$ and for the same reaction time of $4.5 \mathrm{~h}$. These resulted in GNPs decorated with $\mathrm{ZnO}$ microrods (ZnOMRs) as detailed in the following. This procedure, hereinafter referred to as hydrothermal method in static condition, is denoted as HT2.

Growth of ZnO-NRs onto GNPs suspended in aqueous solution by probe sonication method. The growth of ZnO-NRs onto seeded GNPs suspended in aqueous suspension was obtained through the probe sonication method, which is known to be characterized by a higher growth-rate with respect to the hydrothermal method. ${ }^{27}$ In this case the growth reaction step was assisted by probe sonication. The probe sonication of the seeded GNPs suspension in growth solution was performed for 15 min, with ultrasound amplitude set at $45 \%$ of its maximum amplitude in continuous mode. This growth condition is referred in the following as PS.

\subsection{Characterizations}

Samples for field emission scanning electron microscope (FESEM), high resolution transmission electron microscopy (HRTEM), X-ray Photoelectron Spectroscopy (XPS), Auger Electron Spectroscopy (AES), Ultraviolet Photoelectron Spectroscopy (UPS) and photoluminescence (PL) analyses were prepared from the suspensions produced through either hydrothermal or probe sonication method. To this purpose, a concentrated suspension of hybrid nanostructures obtained upon centrifugation (for $30 \mathrm{~min}$ at $3095 \mathrm{~g}$ ) was drop-casted onto cleaned silicon substrates (holey carbon grid coated on a 200 mesh $\mathrm{Cu}$ grids for HR-TEM), with subsequent drying in oven at $120{ }^{\circ} \mathrm{C}$ for 30 min. X-ray diffraction (XRD) was performed on ZNG powders obtained by centrifugation of the ZNG suspension and subsequent drying in oven at $120^{\circ} \mathrm{C}$ for $30 \mathrm{~min}$.

The morphology, homogeneity and density of the produced hybrid nanostructures were observed using a FE-SEM (Zeiss Auriga). Samples were imaged without any metal coating, with an accelerating voltage varying between 3 and $5 \mathrm{keV}$.

The crystalline analysis was carried out using a HR-TEM (FEI TECNAI F20) equipped with a Schottky emitter and operated at 200 $\mathrm{kV}$, by means of high resolution imaging and electron diffraction.
The structural characterization and phase identification was performed by XRD using a Bruker AXS D8-Advance diffractometer, operating in $\theta-\theta$ transmission geometry, equipped with focusing Göbel mirrors along the incident beam, Soller slits on both incident and (radial) diffracted beams, and a PSD VANTEC-1 detector. Samples for XRD were prepared loading the powders into $0.3 \mathrm{~mm}$ diameter borosilicate capillaries that were aligned onto a standard goniometer head. XRD data were collected at room temperature, using $\mathrm{Cu} \mathrm{K} \alpha$ radiation $(\lambda=1.5418 \AA, 40 \mathrm{kV}$ at $40 \mathrm{~mA}$ ), in a $2 \theta$ angular range ranging from $20^{\circ}$ to $145^{\circ}$ with a step size of $0.022^{\circ}$ and $1 \mathrm{~s}$ of counting time. Structural refinements were performed by the Rietveld method using TOPAS v.4.2 (Bruker AXS 2009) software. Starting structural parameters of hexagonal $\mathrm{ZnO}$ (zincite) were taken from Sowa and Ahsbahs. ${ }^{30}$

The chemical composition analysis was performed by XPS using a monochromatized spectrometer Escalab 250Xi (Thermo Fisher Scientific, UK) equipped with a six-channeltron detection system for spectroscopy and a multichannel plate for chemical imaging. The measurements were carried out on the powders deposited onto silicon substrates and placed in an ultra-high vacuum (UHV) chamber, using the $\mathrm{Al} \mathrm{K} \alpha$ X-ray source set to the diameter of $900 \mu \mathrm{m}$, the analyser - at constant pass energy of $20 \mathrm{eV}$ and standard mode of electromagnetic input lenses (about $1 \mathrm{~mm}$ in diameter). Acquired spectroscopic data were processed by the Avantage v.5 software (Thermo Fisher Scientific, UK). UPS measurements were carried out using ultraviolet light source of He I and II. A constant pass energy of $5 \mathrm{eV}$ and 20 eV were used for He I and He II sources, respectively. Chemical images were acquired using $500 \mu \mathrm{m} \mathrm{X}$-ray source, electromagnetic mode of input lenses, $100 \mathrm{eV}$ pass energy of the analyzer and multichannel plate detector.

The crystalline defect analysis was studied through room temperature PL using as excitation source the $266 \mathrm{~nm}$ line of a Nd:YAG pulsed laser (Thomson DIVA), with $10 \mathrm{~ns}$ pulse length and $20 \mathrm{~Hz}$ repetition rate. Energy dispersion of the PL signal was obtained by using a 3648-element linear silicon CCD array, operating in the 200-1100 nm wavelength range. The PL spectra were repeated 16 times on each sample at two different levels of the laser mean power (i.e. 700 and $1300 \pm 100 \mathrm{~W} \mathrm{~m}^{-2}$ ) and the average values were reported versus the emission energy.

\section{Results and discussions}

\subsection{Morphology analysis by FE-SEM}

All the produced samples, with their main morphological characteristics, are listed in Table 1.

The surface morphology observed by FE-SEM of seeded GNPs, produced either by magnetic stirring (sample G1) or by probe sonication (sample G2) of the GNPs suspension in the seed solution is shown in Fig. 1. In both cases, the seed $\mathrm{ZnO}$ nanoparticles are uniformly deposited onto the GNP surfaces, they are quasi spherical in shape and anchored very well to the GNP surfaces. The diameter of the seed nanoparticles in sample G1 (Fig. 1A) is in the range of 10-30 nm, whereas in sample G2 (Fig. 1B) they are slightly smaller, being in the range of 10-20 $\mathrm{nm}$, but with a higher coverage density. Hence, the seed layer deposition assisted by probe sonication, with respect to the one 
Table 1 List of all produced samples and their main morphological characteristics (average values)

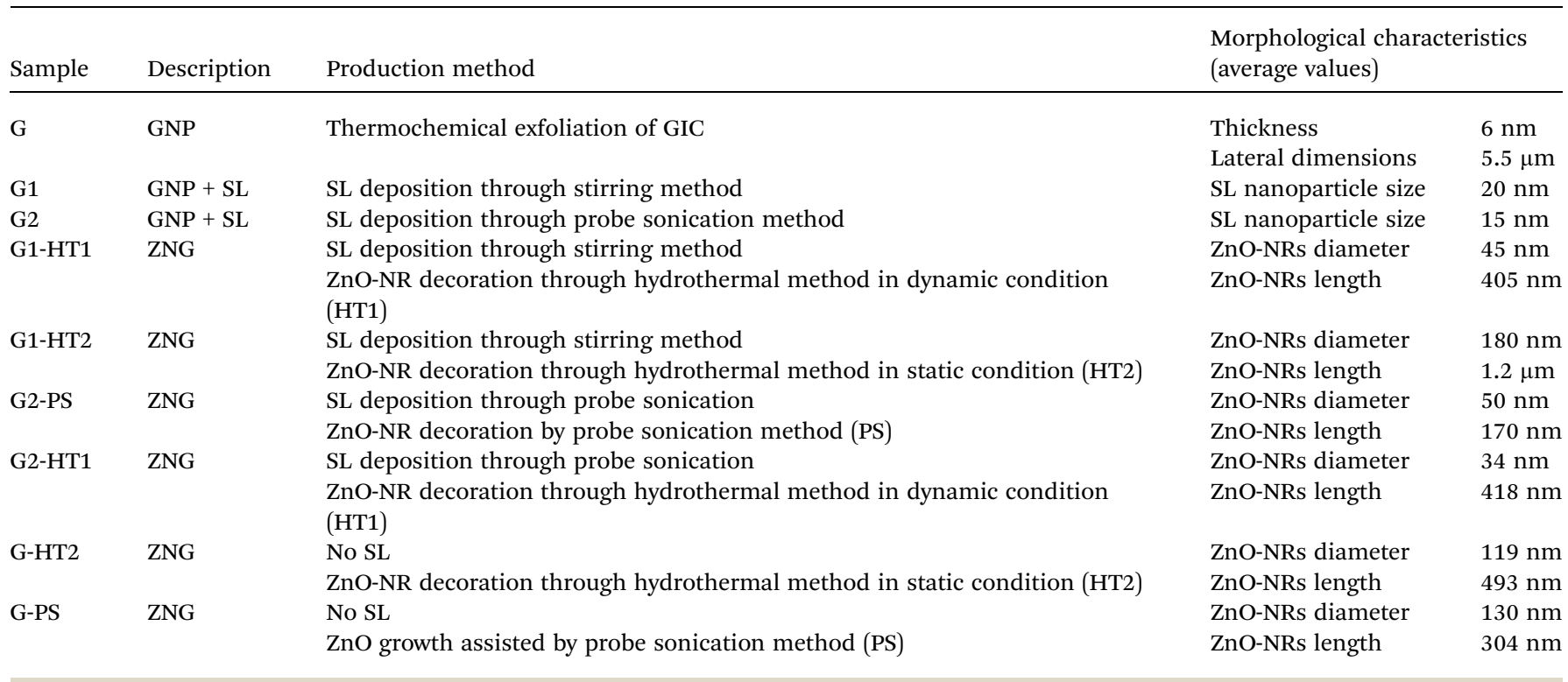

assisted by magnetic stirring, resulted in more densely decorated GNPs with smaller seed nanoparticles.

Fig. 2 shows the morphology of the different samples of Table 1. ZnO-NRs with the highest aspect ratio (i.e. length-todiameter ratio) are obtained either using seeded GNPs produced with magnetic stirring (Fig. 2A and B) or probe sonication (Fig. 2G and $\mathrm{H}$ ), through hydrothermal method under dynamic condition (method HT1). In both cases, the seed particles are acting as nucleation sites of the $\mathrm{ZnO}$ nanostructures, which growth in a flower-like configuration.

Due to the high-density of the growth, ZnO-NRs appear mostly vertically oriented with respect to the GNP surface, uniform in size, with a diameter ranging from 20 to $50 \mathrm{~nm}$ and length ranging from 300 to $500 \mathrm{~nm}$.

Sample G2-HT1 shown in Fig. $2 \mathrm{G}$ and $\mathrm{H}$ is characterized by a higher density of ZnO-NRs with respect to sample G1-HT1 of Fig. 2A and B: this is mainly due to the better coverage of seed nanoparticles produced by the seed-layer deposition assisted by probe-sonication.

Hydrothermal growth onto seeded GNPs carried out in static condition (method HT2) produces ZnO microrods (ZnO-MRs)
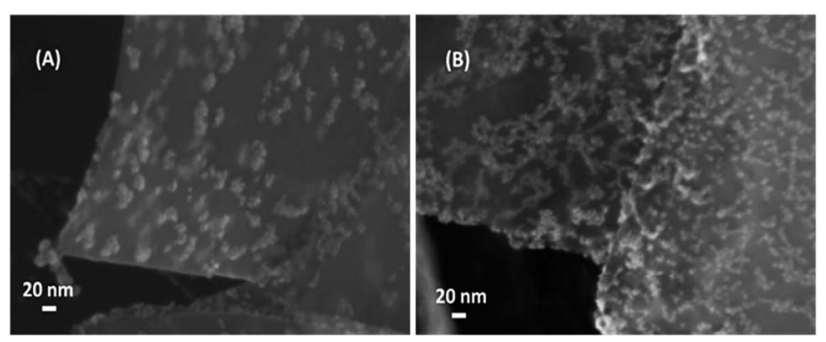

Fig. 1 Electron microscopy images of GNPs with seed-layer: (A) seeded GNPs produced with magnetic stirring of the seed solution (sample G1 in Table 1) and (B) seeded GNPs produced with probesonication of the seed solution (sample G2 in Table 1). having diameter ranging from 100 to $300 \mathrm{~nm}$ and length ranging from $600 \mathrm{~nm}$ to $1.50 \mu \mathrm{m}$ (as shown in Fig. 2C and D), with a lower coverage density. In this case, the flower-like nucleation of ZnO-MRs from the seed nanoparticles is more evident.

The use of PS method during both seed deposition and nanostructure growth originates flower like bunches of $\mathrm{ZnO}$ NRs with a low aspect ratio. These ZnO-NRs have diameter ranging from 40 to $60 \mathrm{~nm}$ and length ranging from 150 to 200 $\mathrm{nm}$ (Fig. 2E and F). It is observed that in this case nanostructure coverage over GNPs surface is not very uniform. The main advantage of this technique is that nanorods growth takes place in few minutes, whereas the hydrothermal method needs several hours.

The role of the SL deposition in the high density growth of ZnO-NRs onto GNPs was assessed by performing the growth in the absence of such layer. The obtained FE-SEM results are shown in the ESI (page 2, Fig. S2 $\dagger$ ). It is evident that in this case it is not possible to achieve a uniform and dense distribution of ZnO-nanostructures over the GNP exposed surfaces. In fact, the growth without SL can be mainly ascribed to the presence of surface defects on the unseeded GNPs, which might act like nucleation centers for the growth. ${ }^{31}$ This confirms the importance of the deposition of the SL onto unsupported GNPs.

Table 2 summarizes the difference of the effects on the size and density of the ZnO-NRs grown onto the graphene nanoplatelets, between hydrothermal method and ultrasonic probe sonication method.

\subsection{Crystalline and structural analysis by HR-TEM and XRD}

The interaction between ZnO-seed particles and GNPs was investigated by HR-TEM analysis. To this purpose, sample G1HT1 of Table 1 was considered, since it is quite representative of all the other samples described. Fig. 3A and B shows HR-TEM images of the ZNGs produced by hydrothermal method in 


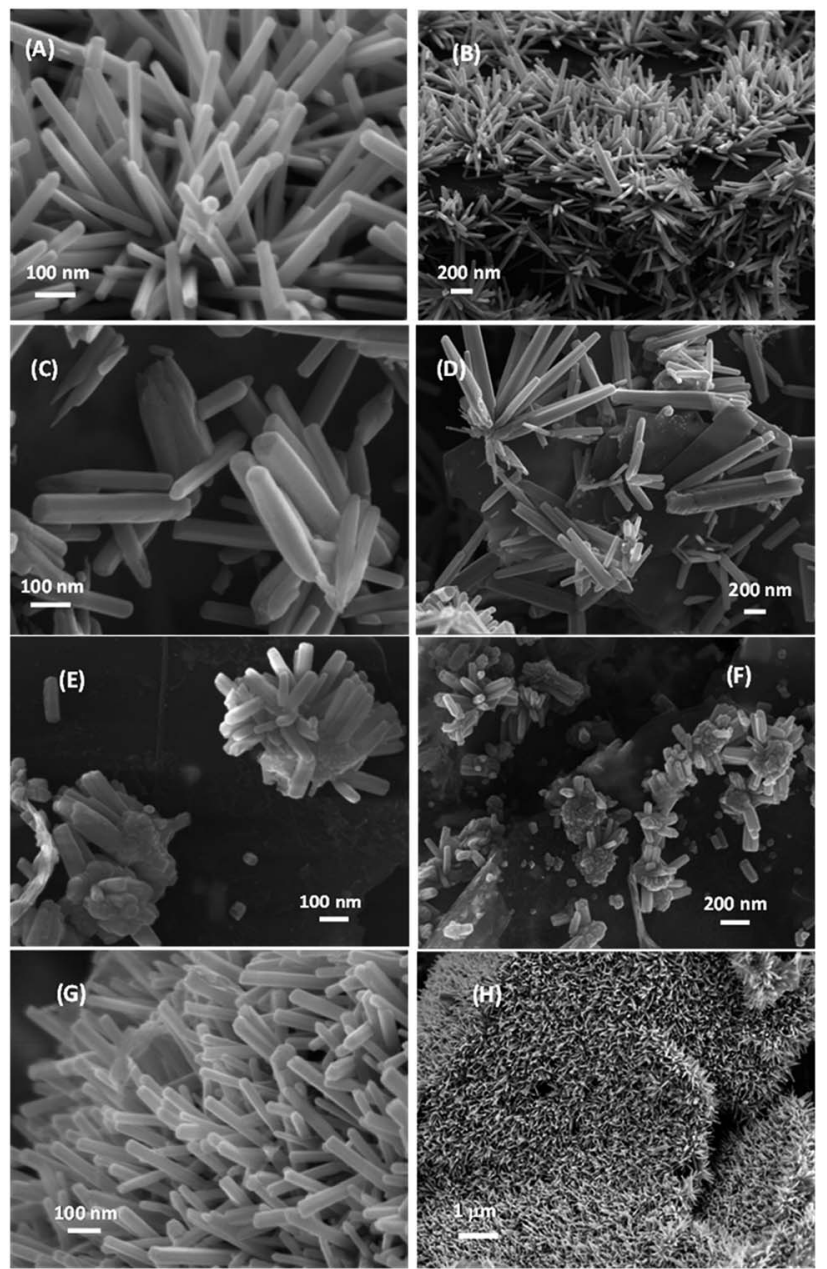

Fig. 2 Electron microscopy images of ZNGs at different magnifications produced starting from (A-D) seeded GNPs obtained through mechanical stirring (sample $\mathrm{G} 1)$ or $(\mathrm{E}-\mathrm{H})$ probe sonication of the seed solution (sample G2): (A and B) sample G1-HT1 obtained by hydrothermal growth in dynamic condition of seeded graphene G1; ( $C$ and D) sample G1-HT2 obtained by hydrothermal growth in static condition of seeded graphene G1; (E and F) sample G2-PS obtained by probe sonication of seeded graphene G2; ( $G$ and H) sample G2-HT1 obtained by hydrothermal growth in dynamic condition of seeded graphene $G 2$.

dynamic condition, starting from seeded GNPs produced by SL deposition assisted by magnetic stirring (sample G1-HT1). Fig. 3A clearly demonstrates that the planar and transparent graphene sheets are decorated with rod-like $\mathrm{ZnO}$ nanostructures. Fig. 3B highlights the intimate physical interaction between graphene and $\mathrm{ZnO}$-seed particle. It can also be seen from Fig. 3B that the ZnO-NRs are radially evolved from these seed particles, which are anchored over the unsupported graphene sheets. Thereby, these seed particles play an important role in the growth mechanism of ZnO-NRs over the GNPs with high density. Selected area electron diffraction (SAED) pattern shown in the inset of Fig. 3B, with highlighted the (100) and (110) reflections of $\mathrm{ZnO}$ as well as the (002) of graphene, confirms that the crystalline $\mathrm{ZnO}$ nanorods lie on the graphene sheets.

Fig. 4 shows the diffraction patterns of the produced ZNGs, compared with one of pristine GNPs. The sharp and intense peaks of the patterns clearly indicate the highly crystalline nature of the ZNGs. Moreover, peaks positions and intensities agree with those published in the Joint Committee on Powder Diffraction Standards (JCPDS) card 036-1451 for the hexagonal polymorph (zincite) of $\mathrm{ZnO}$.

Detailed structural information on the analyzed samples is reported in the ESI (page 4, Table $\mathrm{S} 1 \dagger$ ). The cell parameters and $\langle\mathrm{Zn}-\mathrm{O}\rangle$ bond distance of 1.978-1.979 $\AA$ obtained for all the samples is in perfect agreement with the findings of Sowa and Ahsbahs. ${ }^{30}$

\subsection{Chemical purity analysis by XPS}

The surface chemical composition of the produced ZNG samples was analyzed by XPS in order to detect traces of any impurities and to assess the homogeneity of the surface composition. For comparison, the same analysis was carried out on the reference sample containing pristine GNPs. All measurements were carried out at three different points on each sample. These three points on each sample were found to be very similar to each other, which indicate that $\mathrm{ZnO}$ is homogeneously distributed on the surface of the investigated samples.

In all cases, zinc is always present in the form of $\mathrm{ZnO}$, i.e. in $\mathrm{Zn}^{+2}$ chemical state, which is confirmed by the binding energy (BE) value of $\mathrm{Zn} 2 \mathrm{p}_{3 / 2}$ peak at $\mathrm{BE} \approx 1022.5 \mathrm{eV}$ and by the position of Auger $\mathrm{Zn}$ LMM peak at kinetic energy $\mathrm{KE} \approx 987.3 \mathrm{eV} \cdot{ }^{32,33} \mathrm{In}$ addition, the value of modified Auger parameter $(\alpha \approx 2009.8$ $\mathrm{eV}$ ) corresponds to the $\mathrm{Zn}(+2)$ chemical state. ${ }^{33}$ The spectra of $\mathrm{Zn} 2 \mathrm{p}$ and $\mathrm{Zn}$ LMM regions for the ZNG samples are shown in Fig. 5A and B respectively.

The 01s spectra, including the peak fitting, of the hybrid ZNG samples and of the reference sample are presented in Fig. 6.

The O1s spectra of all the ZNG samples consist of three components indicated as A, B and C. The first component A is attributed to $\mathrm{O}^{2-}$ ions in the wurtzite structure of $\mathrm{ZnO},{ }^{47}$ while the second one is attributed to the hydroxyl groups $\mathrm{OH}^{-}$and/or $\mathrm{CO}_{3}$ radicals and the third one is assigned to adsorbed $\mathrm{H}_{2} \mathrm{O}$ molecules. ${ }^{32,34}$

It should be noted that in the reference sample the first component (oxide) is completely absent, while the other two components $\left(\mathrm{OH}^{-}\right.$and $\left.\mathrm{H}_{2} \mathrm{O}\right)$ are very low. Whereas in all other ZNGs samples noticeable variation in the atomic percent of O1s components is observed, ascribed to the respective variance in the growth conditions. Although samples G1-HT1 and G1-HT2 are prepared by same method but the growth solution dynamics are different, resulting in high variation in the atomic percent of $\mathrm{OH}^{-}$groups and their effect on crystalline quality is assessed by PL and discussed in Section 3.5.

For all produced ZNG samples, the values of asymmetric C1s peak at $\mathrm{BE} \approx 284.4 \mathrm{eV}$ and the valence band $(\mathrm{VB})$ spectra, starting at $2.5 \mathrm{eV}$ above Fermi level (typical for graphene ${ }^{34}$ ), indicate the presence of graphene. In the reference sample, carbon is present in the form of graphite, because even if the C1s peak remains at $\mathrm{BE} \approx 284.4 \mathrm{eV}$, the $\mathrm{VB}$ spectrum starts at the Fermi level, i.e. at $\mathrm{BE}=0 \mathrm{eV}$ (typical for graphite). The C1s 
Table 2 Summary of the main differences of the effects on the size and density of ZnO-NR grown over GNPs, between hydrothermal and probe sonication methods

\begin{tabular}{lll}
\hline Process & Hydrothermal method & Probe sonication method \\
\hline \multirow{2}{*}{ Seed layer (SL) depostion over GNPs } & Small seed particles & Very small seed particles \\
& Good coverage and good density & Best coverage and highest density \\
& SL coating reaction $(30 \mathrm{~min})$ & Faster SL coating reaction $(15 \mathrm{~min})$ \\
& Nanorods by dynamic condition & Flower like bunches of nanorods \\
& Microrods by HT2 & \\
& Vertical orientation for nanorods & Vertical orientation \\
& Random orientation for microrods & \\
& Narrow size range for nanorods & Narrow size range \\
& Wide size range for microrords & \\
& High aspect ratio & Low aspect ratio \\
& Higher coverage density by HT1 & Poor coverage density \\
& Lower coverage density by HT2 & Faster growth rate (growth reaction for 15 min)
\end{tabular}

and VB spectra of the samples are reported in Fig. 7A and B respectively. A high peak immediately after the valence band at $\mathrm{BE} \approx 11 \mathrm{eV}$ is attributed to $\mathrm{Zn} 3 \mathrm{~d}$ core level and is absent in the reference sample without $\mathrm{ZnO}$ (see Fig. 7B). The main difference between the samples is the variation in the atomic concentrations of $\mathrm{C}$ and $\mathrm{Zn}$. The relative reduction of carbon amount corresponds to the higher coverage of $\mathrm{ZnO}$ on the surface. In the order of higher $\mathrm{C}$ content, the samples can be listed as: G2-PS > G1-HT2 > G1-HT1. This indicates that the sample G1-HT1 contains the best coverage of $\mathrm{ZnO}$, which can be listed in the following order: G1-HT1 > G1-HT2 > G2-PS. These results indicate that the preparation methods ensured the production of pure oxide (ZnO) using all three methods G1-HT1, G1-HT2 and G2-PS, as the $\mathrm{Zn}$ and $\mathrm{O}$ were the only elements detected on the surface of graphene. The chemical composition and chemical states of elements for all the samples are reported in the ESI (page 4, Table S $2 \dagger$ ).

Furthermore, the XPS chemical images were acquired over the area of $100 \times 100 \mu \mathrm{m}^{2}$ on all the samples and demonstrated that the $\mathrm{Zn} 2 \mathrm{p}$ signal is homogeneous and densely distributed all over the surface as shown in the ESI (page 5, Fig. S3†).

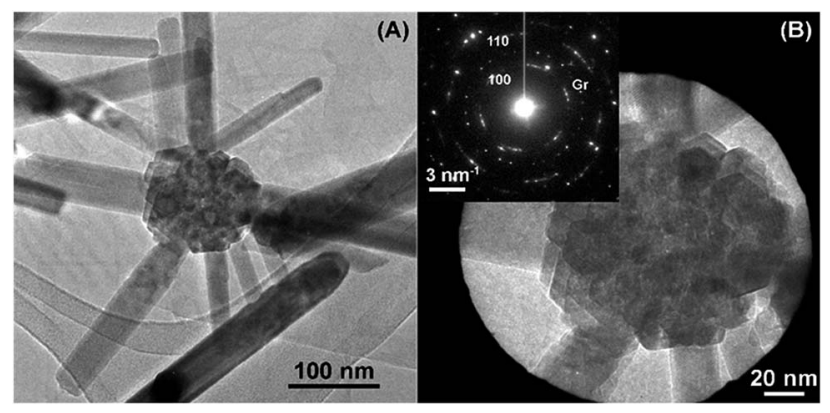

Fig. 3 (A) HR-TEM images of ZNGs produced through hydrothermal growth in dynamic condition starting from seeded GNP obtained by seed-layer deposition assisted by magnetic stirring, and (B) high magnification image showing the intergration of seed particle and GNP. In the inset the SAED pattern shows the presence of $\mathrm{ZnO}$ crystals and graphene $(\mathrm{Gr})$.

\subsection{Valence band analysis by UPS}

The work function (WF) values of the hybrid ZNG samples were analyzed by UPS in order to assess the doping and homogeneity of the composition. Fig. 8A shows the valence band spectra acquired by He I source to calculate the WF values. WF of the samples was calculated by subtracting the spectra cut-off values from the energy of the He I source which is equal to $21.2 \mathrm{eV}$. The values of WF were the following: for G1-HT1 sample 21.2-16.2 = $5 \mathrm{eV}$; for G1-HT2 sample 21.2-16.2 = 5 eV; for G2-PS sample $21.2-16.2=5 \mathrm{eV}$; for reference sample $21.2-16=5.2 \mathrm{eV}$. All the ZNG samples have similar values of WF equal to $5 \mathrm{eV}$, which corresponds to the value of $\mathrm{ZnO}$ from the literature. ${ }^{35-37}$ The reference sample has the value of the WF of $5.2 \mathrm{eV}$, which is in agreement to the value of graphite from the literature. ${ }^{33-35}$ This confirms doping and that the state of zinc is $\mathrm{ZnO}$.

Valence band (VB) spectra were also acquired by using He II source with a higher energy of $40 \mathrm{eV}$, as shown in Fig. 8B. These spectra confirm the conclusions derived from VB spectra acquired by using X-ray source as shown in Fig. 7. In the samples G1-HT1, G1-HT2 and G2-PS, the signal starts approximately at $\mathrm{BE}=2.5 \mathrm{eV}$, which is a typical behavior for graphene. In the reference sample, the spectrum started at $0 \mathrm{eV}$, which is typical for graphite. In all three ZNG samples, the core-level peak of $\mathrm{Zn} 3 \mathrm{~d}$ is identified at approximately $\mathrm{BE}=11 \mathrm{eV}$, which

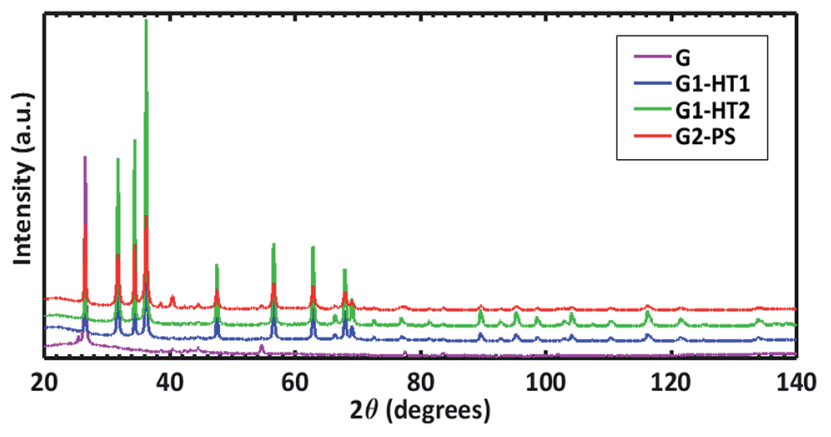

Fig. 4 XRD patterns of pristine GNPs (sample G of Table 1) and of the different types of ZNGs obtained in different growth conditions experienced in this study: samples G1-HT1, G1-HT2, and G2-PS. 

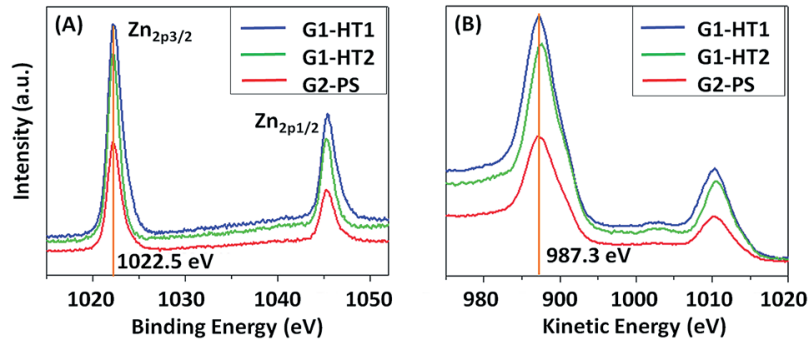

Fig. 5 (A) Zn2 $p_{3 / 2}$ and (B) Zn LMM regions of the ZNGs produced in the different growth conditions experienced in this study: samples G1HT1, G1-HT2, and G2-PS.

confirms that in them GNPs are being doped successfully with ZnO. In sample G1-HT1, the intensity of Zn3d peak is higher than in G1-HT2 and G2-PS samples, i.e. is confirmed our earlier conclusion that G1-HT1 sample has more homogeneous distribution and higher amount of $\mathrm{ZnO}$ than other samples.

\subsection{Crystalline defects analysis by photoluminescence}

The effect on the ZNG luminescence properties of the static- or dynamic-growth condition was studied via PL spectroscopy. The obtained results were compared with the ones of the XPS analysis in order to assess the effect of observed differences in the atomic percent of $\mathrm{OH}^{-}$groups of samples produced applying method HT1 or HT2 (Fig. 6A and B).

Fig. 9 shows the PL spectra of samples G1-HT1 and G1-HT2, produced by dynamic and static methods, respectively. Both samples exhibited PL spectra which are typical of ZnO nanostructures, consisting of a strong UV excitonic emission at $\sim 3.26 \mathrm{eV}$ and a weak and broad VIS emission with its maximum at about $2.13 \mathrm{eV}$. A similar spectrum is obtained when low lasing power is used and it is shown in ESI (page 5, Fig. S4 $\dagger$ ). The UV/ VIS peak intensity ratio is used to evaluate the crystal quality, i.e. the absence of electronic defects which may act as recombination centers for charge carriers and quench the excitonic

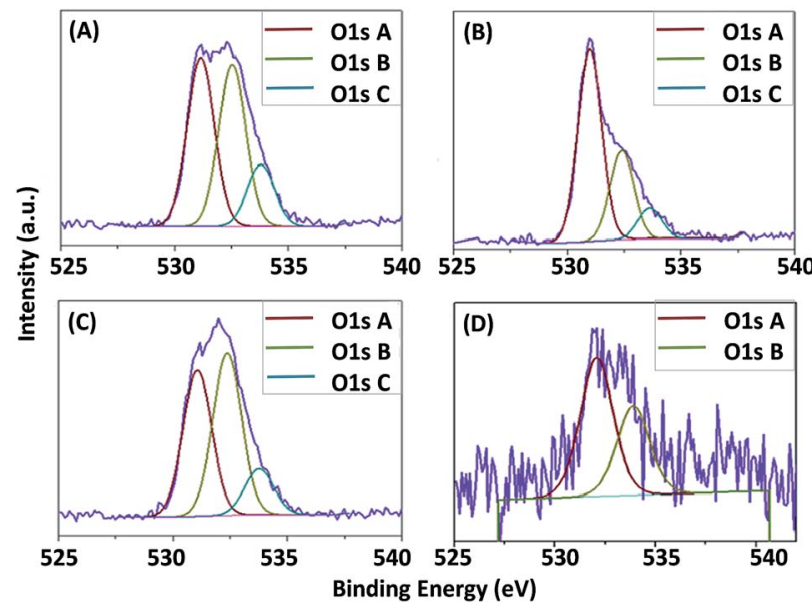

Fig. 6 O1s spectra of the ZNGs produced in the different growth conditions experienced in this study: (A) G1-HT1, (B) G1-HT2, (C) G2PS, and (D) reference pristine GNPs.

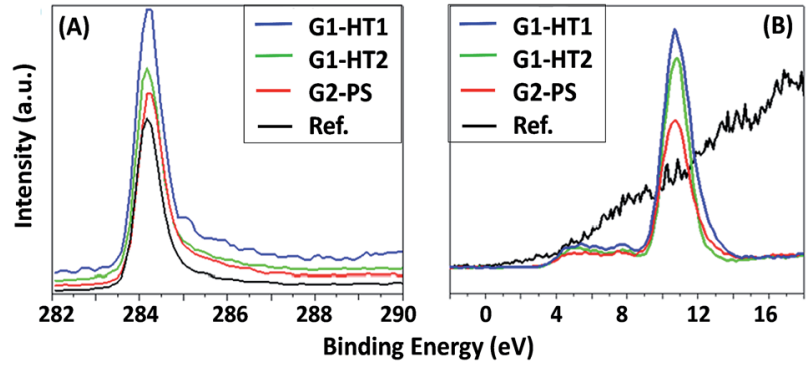

Fig. 7 (A) C1s and (B) XPS valence band spectra for the produced ZNGs (samples G1-HT1, G1-HT2 and G2-PS) and pristine GNPs (sample G).

peak. ${ }^{38,39}$ As evident from Fig. 9, the intensity of UV emission is much higher for sample G1-HT2 than G1-HT1, despite there is not any difference in the weak VIS emission. This suggests that the concentration of defects is lower in samples grown in static hydrodynamic conditions. This observation is consistent with the evidenced differences in the O1s components of XPS spectra (Fig. 6A and B), since a more intense O1s B line is known to be associated to the presence of various defects, responsible for damping the UV PL emission. ${ }^{40,41}$

Erki Kärber et al. $(2011)^{42}$ reported the various factors affecting the PL of $\mathrm{ZnO}$ nanorods deposited by chemical spray pyrolysis including the spray rate. They observed that by increasing the spray rate from $1.2 \mathrm{ml} \mathrm{min}^{-1}$ to $6.2 \mathrm{ml} \mathrm{min}^{-1}$ the intensity of UV excitonic peak is diminished and slightly shifted, due to the increase of the growth rate (which is consistent with what we observed, i.e. growth rate of sample G1-HT2 is lower than of G1-HT1). Hatim Alnoor et al. (2015) ${ }^{40}$ reported the influence of varying the duration of the precursor solution stirring (prior to the growth) on the concentration and spatial distributions of deep level defects in ZnO-NRs, attributed to variation in the concentration of the oxygen-deficiency. T. Dai et al. (2014) ${ }^{41}$ reported on the enhancement of PL emission of ZnO sol-gel nanoparticles by thermal annealing, resulting from the elimination of surface defects associated to adsorbed $\mathrm{OH}$ bonds. However, to the best of our knowledge, no particular study was performed to investigate the dynamics of nutrient solution during the growth and their effect on PL emission.

Actually during the solution growth, the presence of $\mathrm{OH}^{-}$ which further reacts with $\mathrm{Zn}^{+2}$ and later (during the formation

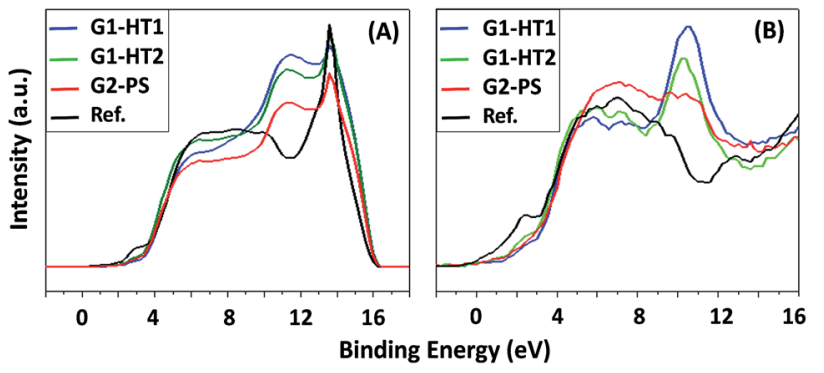

Fig. 8 UPS valence band spectra obtained by using; (A) $\mathrm{He} \mathrm{I}$ and (B) $\mathrm{He}$ II sources. Work function was calculated from the cut-off in $\mathrm{He}$ I spectra. 
of $\mathrm{ZnO}$ nanostructures) promotes the growth mechanism, is higher for G1-HT1 than in G1-HT2. This favors the formation of smaller nanostructures (as explained by DLA theory ${ }^{43}$ ), as well as the presence of surface adsorbed $\mathrm{OH}^{-}$groups (i.e. $\mathrm{ZnO}(\mathrm{OH})$, due to the excess solvent capped on the surface of $\mathrm{ZnO}^{\mathbf{4 1}}$ ). Both these effects may be responsible for a higher number of oxygenrelated surface defects, either due to a larger mass specific area, either due to higher concentration of surface adsorbed $\mathrm{OH}^{-}$ groups.

XRD analysis already demonstrated that all the samples are perfectly crystalline. Therefore, the relatively lower PL emission evinced in sample G1-HT1 can be more likely ascribed to the presence of surface defects than to structural (bulk) ones. Although a significant variation in the oxygen-component is observed for static and dynamic conditions which affect the optical quality, the ZNG nanostructures produced are of perfectly crystalline and chemically pure.

\subsection{Potential applications of ZNGs with controlled size and density of ZnO-NRs}

Over the last decade, vertically aligned ZnO-NRs has been intensively studied due to their remarkable piezoelectric properties and potential for various applications. The improvement and control in the density and size of ZnO-NRs grown onto graphene sheet has a direct beneficial effect on the heterostructured nanogenerator performance. ${ }^{5}$ The facile fabrication process developed in this study suggests that $\mathrm{ZnO}$-graphene heterostructures with tailored morphology would improve the performance of electrical and optoelectrical device, such as touch pad, pressure sensor, biosensor and dye-sensitized solar cells. ${ }^{5,22,23}$

In a recent study, ${ }^{44}$ it has been shown how the control of size and morphology of ZnO-NRs grown onto graphene oxide sheets contributes to an enhancement of the photocatalytic properties of these material with respect to UV radiation. The comparison of the photocatalytic performances of different types of graphene-ZnO hybrids in photodegradation of methylene blue was also reported. ${ }^{\mathbf{4 4}}$

A new promising field of application of the ZNGs developed in this study is related to their antibacterial and antibiofilm properties. In previous studies, the authors have demonstrated the stronger potential of $\mathrm{ZnO}-\mathrm{NRs}$ as antimicrobial agent with

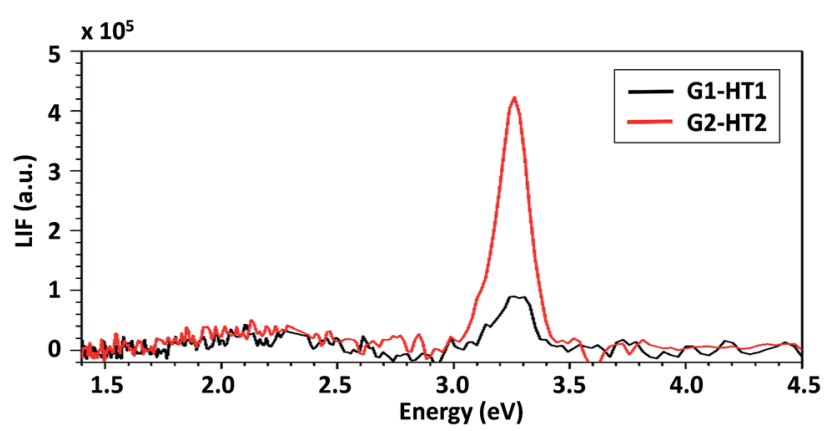

Fig. $9 \mathrm{PL}$ spectra of samples G1-HT1 and G1-HT2; incident laser power $=1300 \mathrm{~W} \mathrm{~m}^{-2}$. respect to other types of $\mathrm{ZnO}$ nanostructures (like nanoparticles or microrods), due to their particular shape and size. In fact, ZnO-NRs, having diameter in the range $40-50 \mathrm{~nm}$ behave as nano-needles against pathogens, ${ }^{8}$ although they are characterized by very low cytotoxicity as shown through in vitro studies. ${ }^{45}$ At the same time, GNPs have demonstrated relevant antimicrobial effect against both Gram-negative ${ }^{46}$ and Gram-positive ${ }^{47}$ bacteria, due to the direct mechanical damage induced on the cell wall through their sharp edges and the wrapping effect. However, the use of GNPs as antibacterial material has some limitations in those applications in which their characteristic grey color is a problem, like in dental use or in cultural heritage. Weak antimicrobial efficacy of GNPs was also observed against spherical-shaped bacteria with relatively small size, like $S$. aureus or $S$. mutans,${ }^{47}$ offering a limited interaction surface with the 2D-nanostructures, having lateral dimensions from $500 \mathrm{~nm}$ up to a few microns. ZNGs, with high-density of ZnO-NRs less than $50 \mathrm{~nm}$ in diameter, allow to combine the antimicrobial and antibiofilm properties of both ZnO-NRs and GNPs and, at the same time they enable to overcome the color limitation of GNPs thanks to the optical properties of $\mathrm{ZnO}$.

\section{Conclusions}

Facile versatile aqueous methods to decorate unsupported GNPs with shape-controlled ZnO-NRs have been demonstrated. Different hydrodynamic conditions during the ZNG synthesis are experimented, on the basis of two distinct growth methods (i.e. the hydrothermal method and probe sonication), starting from seeded GNPs suspended in aqueous solution.

Hydrothermal growth performed in dynamic condition resulted in a dense growth of mostly vertically oriented crystalline wurtzite $\mathrm{ZnO}$-NRs having diameter of $\sim 45 \mathrm{~nm}$ and length of $\sim 405 \mathrm{~nm}$. On the contrary, HT synthesis in static condition resulted mainly in the growth of flower-like bunches of $\mathrm{ZnO}$ MRs with a lower coverage density, average diameter of $\sim 180$ $\mathrm{nm}$ and length up to 1-1.5 $\mu \mathrm{m}$.

Probe sonication growth method is demonstrated to be a faster process producing flower-like bunches of ZnO-NRs with low aspect ratio (i.e. average diameter of $\sim 50 \mathrm{~nm}$ and length of $\sim 170 \mathrm{~nm}$ ).

In conclusions, the highest growth density with utmost homogeneity of nanorods is obtained onto GNPs seeded by probe sonication method and growth carried out by hydrothermal method in dynamic condition. This type of ZNGs has very promising applications as building block in piezoelectric nanogenerator or as antimicrobial/antibiofilm device.

Although solution dynamics affected the UV luminescence, XRD and XPS investigations confirmed that all the produced ZNGs are characterized by high crystallinity and purity. UPS work function analysis suggested a possible doping effect of ZnO-NRs grown over GNPs.

\section{Acknowledgements}

This study was performed under the project PON03PE_00214_1. 


\section{References}

1 X. Huang, X. Qi, F. Boeyab and H. Zhang, Chem. Soc. Rev., 2012, 41, 666.

2 L. Paliotta, G. De Bellis, A. Tamburrano, F. Marra, A. Rinaldi, S. K. Balijepalli, S. Kaciulis and M. S. Sarto, Carbon, 2015, 89, 260.

3 H. Chang and H. Wu, Energy Environ. Sci., 2013, 6, 3483.

4 C. Hsieh, C. Lin, Y. Chen and J. Lin, Electrochim. Acta, 2013, 111, 359.

5 D. M. Shin, E. L. Tsege, S. H. Kang, W. Seung, S. W. Kim, H. K. Kim, S. W. Hong and Y. H. Hwang, Nano Energy, 2015, 12, 268-277.

6 S. Xu and Z. L. Wang, Nano Res., 2011, 4, 1013.

7 I. Rago, C. R. Chandraiahgari, M. P. Bracciale, G. De Bellis, E. Zanni, M. C. Guidi, D. Sali, A. Broggi, C. Palleschi, M. S. Sarto and D. Uccelletti, RSC Adv., 2014, 4, 56031.

8 H. Chang, Z. Sun, K. Y. F. Ho, X. Tao, F. Yan, W. M. Kwok and Z. Zheng, Nanoscale, 2011, 3, 258.

9 R. Zou, G. He, K. Xu, Q. Liu, Z. Zhang and J. Hu, J. Mater. Chem. A, 2013, 1, 8445.

10 X. Dong, Y. Cao, J. Wang, M. B. Chan-Park, L. Wang, W. Huanga and P. Chen, RSC Adv., 2012, 2, 4364.

11 T. Song, J. W. Choung, J. G. Park, W. I. Park, J. A. Rogers and U. Paik, Adv. Mater., 2008, 20, 4464.

12 Z. Chen, N. Zhang and Y. Xu, CrystEngComm, 2013, 15, 3022.

13 L. Zhang, X. Liu, Z. Lian, X. Wang, G. Shen, D. Shen and Q. Yan, J. Mater. Chem. C, 2014, 2, 3965.

14 A. G. D'Aloia, F. Marra, A. Tamburrano, G. De Bellis and M. S. Sarto, Carbon, 2014, 73, 175-184.

15 G. De Bellis, A. Tamburrano, A. Dinescu, M. L. Santarelli and M. S. Sarto, Carbon, 2011, 49, 4291-4300.

16 C. M. Lieber, Sci. Am., 2001, 285, 50.

17 Y. Ding, P. X. Gao and Z. L. Wang, J. Am. Chem. Soc., 2004, 126, 2066.

18 P. Yang, H. Yan, S. Mao, R. Russo, J. Johnson, R. Saykally, N. Morris, J. Pham, R. He and H.-J. Choi, Adv. Funct. Mater., 2002, 12, 323.

19 B. Liu and H. C. Zeng, J. Am. Chem. Soc., 2003, 125, 4430.

20 C. R. Chandraiahgari, G. De Bellis, P. Ballirano, S. K. Balijepalli, S. Kaciulis, L. Caneve, F. Sarto and M. S. Sarto, RSC Adv., 2015, 5, 49861.

21 J. Ding, S. Zhu, T. Zhu, W. Sun, Q. Li, G. Wei and Z. Su, RSC Adv., 2015, 5, 22935.

22 R. Chung, Z. Lin, P. K. Yang, K. Lai, S. F. Jen and P. W. Chiu, Nanoscale Res. Lett., 2013, 8, 350.

23 Y. J. Kim, Hadiyawarman, A. Yoon, M. Kim, G. C. Yi and C. Liu, Nanotechnology, 2011, 22, 245603.

24 M. Khenfouch, M. Baïtoul and M. Maaza, Opt. Mater., 2014, 36, 27.
25 J. Fan, T. Li, H. Heng, B. Markovic and I. Djerdj, Acta Chim. Slov., 2015, 62, 902.

26 L. Vayssieres, Adv. Mater., 2003, 15, 465.

27 T. O. Okyay, R. K. Bala, H. N. Nguyen, R. Atalay, Y. Bayam and D. F. Rodrigues, $R S C A d v$. , 2015, 5, 2568.

28 G. Cao, Nanostructures and Nanomaterials: Synthesis, Properties and Applications, Imperial College Press, London, UK, 2004.

29 L. E. Greene, M. Law, H. D. Tan, M. Montano, J. Goldberger, G. Somorjai and P. Yang, Nano Lett., 2005, 5, 1231.

30 H. Sowa and H. Ahsbahs, J. Appl. Crystallogr., 2006, 39, 169. 31 J. M. Lee, Y. B. Pyun, J. Yi, J. W. Choung and W. Il Park, J. Phys. Chem. C, 2009, 113, 19134.

32 S. Kaciulis, L. Pandolfi, E. Comini, G. Faglia, M. Ferroni, G. Sberveglieri, S. Kandasamy, M. Shafiei and W. Wlodarski, Surf. Interface Anal., 2008, 40, 575.

33 S. Kaciulis, G. Mattogno, A. Galdikas, A. Mironas and A. Setkus, J. Vac. Sci. Technol., A, 1996, 14, 3164.

34 S. Kaciulis, A. Mezzi, P. Calvani and D. M. Trucchi, Surf. Interface Anal., 2014, 46, 966.

35 Q. Zhao, X. Y. Xu, X. F. Song, X. Z. Zhang, D. P. Yu, C. P. Li and L. Guo, Appl. Phys. Lett., 2006, 88, 033102.

36 J. O. Hwang, D. H. Lee, J. Y. Kim, T. H. Han, B. H. Kim, M. Park, K. No and S. O. Kim, J. Mater. Chem., 2011, 21, 3432.

37 W. Song, S. Y. Kwon, S. Myung, M. W. Jung, S. J. Kim, B. K. Min, M. A Kang, S. H. Kim, J. Lim and K. Seok An, Sci. Rep., 2014, 4, 4064.

38 L. Wu, Y. Wu, X. Pan and F. Kong, Opt. Mater., 2006, 28, 418. 39 A. B. Djurisic, Y. H. Leung, K. H. Tam, L. Ding, W. K. Ge, H. Y. Chen and S. Gwo, Appl. Phys. Lett., 2006, 88, 103107.

40 H. Alnoor, C. O. Chey, G. Pozina, X. Liu, V. Khranovskyy, M. Willander and O. Nur, AIP Adv., 2015, 5, 087180.

41 T. Dai, W. Hsu and H. Hsu, Opt. Express, 2014, 22, 27170.

42 E. Kärber, T. Raadik, T. Dedova, J. Krustok, A. Mere, V. Mikli and M. Krunks, Nanoscale Res. Lett., 2011, 6, 359.

43 G. Cao and Y. Wang, Nanostructures and Nanomaterials: Synthesis, Properties and Applications, Imperial College Press, London, UK, 2004.

44 E. Rokhsat and O. Akhavan, Appl. Surf. Sci., 2016, 371, 590. 45 E. Zanni, S. De Palma, C. R. Chandraiahgari, G. De Bellis, S. Cialfi, C. Talora, C. Palleschi, M. S. Sarto, D. Uccelletti and P. Mancini, Mater. Lett., 2016, 179, 90.

46 E. Zanni, G. De Bellis, M. P. Bracciale, A. Broggi, M. L. Santarelli, M. S. Sarto, C. Palleschi and D. Uccelletti, Nano Lett., 2012, 12, 2740.

47 I. Rago, A. Bregnocchi, E. Zanni, A. G. D'Aloia, F. De Angelis, M. Bossu, G. De Bellis, A. Polimeni, D. Uccelletti and M. S. Sarto, 2015 IEEE 15th International Conference on Nanotechnology (IEEE-NANO), Rome, 2015, pp. 9-12. 